\title{
Tiled Fuzzy Hough Transform for Crack Detection
}

\author{
Kanapathippillai Vaheesan ${ }^{\mathrm{a}}$, Chanjief Chandrakumar ${ }^{\mathrm{b}}$, Senthan Mathavan ${ }^{{ }^{*} \mathrm{c}}$ \\ Khurram Kamal $^{\mathrm{d}}$, Mujib Rahman ${ }^{\mathrm{e}}$, Amin Al-Habaibeh ${ }^{\mathrm{c}}$ \\ ${ }^{a}$ Huawei Technologies, Sri Lanka; ${ }^{b}$ Department of Production Engineering, University of \\ Peradeniya, Sri Lanka; ${ }^{\circ}$ School of Architecture, Design and the Built Environment, Nottingham \\ Trent University, UK; ${ }^{\mathrm{d}}$ College of Electrical and Mechanical Engineering, National University of \\ Sciences and Technology, Pakistan; ${ }^{\mathrm{e} D e p a r t m e n t ~ o f ~ M e c h a n i c a l, ~ A e r o s p a c e ~ a n d ~ C i v i l ~ E n g i n e e r i n g, ~}$ \\ Brunel University, UK
}

\begin{abstract}
Surface cracks can be the bellwether of the failure of any component under loading as it indicates the component's fracture due to stresses and usage. For this reason, crack detection is indispensable for the condition monitoring and quality control of road surfaces. Pavement images have high levels of intensity variation and texture content, hence the crack detection is difficult. Moreover, shallow cracks result in very low contrast image pixels making their detection difficult. For these reasons, studies on pavement crack detection is active even after years of research. In this paper, the fuzzy Hough transform is employed, for the first time to detect cracks on any surface. The contribution of texture pixels to the accumulator array is reduced by using the tiled version of the Hough transform. Precision values of $78 \%$ and a recall of $72 \%$ are obtaining for an image set obtained from an industrial imaging system containing very low contrast cracking. When only high contrast crack segments are considered the values move to mid to high $90 \%$.
\end{abstract}

Keywords: Crack, Hough transform, fuzzy logic, pavement, road, defect

\section{INTRODUCTION}

A nation's development is directly and indirectly influenced by its highway network. Road networks are recognized as one of the key man-made infrastructures and pave-ments are the primary elements. Frequent health monitoring surveys are vital in gath-ering information about pavement conditions. Based on the evaluated severity from the surveys, maintenance decisions are taken to rehabilitate if required. The inspection surveys have been performed manually for a great deal of the twentieth century.

However, manual surveys are not feasible anymore due to the sheer volume of roads. Furthermore, the difficulty is manifold given that the capacity of every road in a net-work is used to the full, necessitating more than frequent inspection to maintain those to a high standard. This increased inspection frequency is unthinkable with human inspection given the shortage of skilled people. In addition, manual highway surveys are prone to human subjectivity. An automated survey, on the other hand, can be fast, accurate and precise, when designed and validated aptly.

Cracks are one of the most common defects encountered in roads. The fracture of the pavement surface lead to cracking. These cracks directly influence on the loss of load-spreading and water-resistant capacity. These detrimental effects then speed up the deterioration process of pavement surface. If these catastrophic cracks are untreated, then the consequences become more severe and these cracks transform into potholes, deform the road and sometimes even produce differential settlement of road [1].

Crack detection in pavement images, such as the one shown in Figure 1, has been an active area of research area for almost the last two decades. However, highways authorities exercise caution in adopting any of the proposed methodologies and as such the research is very active to date [2-3]. This paper presents a novel methodology for crack detection by the use of fuzzy Hough transform. While the line detection capabilities are utilized, additional flexibility is provided by the fuzzified version allowing for the possibility of 'crack' pixels to be situated outside an exact straight line to be incorporated into the detection.

*s.mathavan@ieee.org; phone +31 686396969 

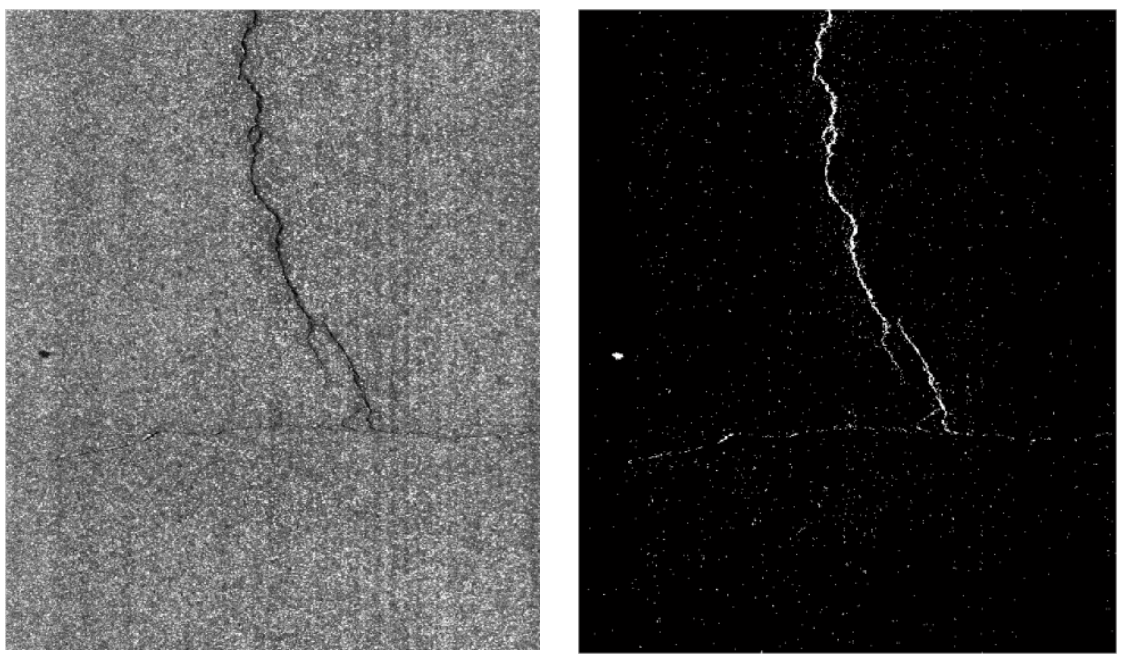

Figure 1. A pavement image captured by the LRIS system of Pavemetrics and its thresholded (and inverted) image.

\section{LITERATURE REVIEW}

An initiation into the area can be obtained from the research conducted by Chen and Miyojim [4] on the techniques applied for the development of automatic pavement distress detection and classification systems. A back propagation neural network is used to identify crack pixels. Furthermore, the authors have proposed a classification method whereby cracks are classified as alligator, transverse and longitudinal. Cheng et.al, in their research [5], proposed a novel technique to pavement crack detection using Fuzzy Set Theory. They compared the darkness of the pixels with the surroundings and mapped the fuzzified images to the crack domain. They then checked the connectivity of the darker pixels in order to reduce pixels lacking connectivity and thereby producing clusters that are eventually detected as crack segments.

In one approach, by classifying neighbors into smooth areas, is utilized by $\mathrm{Hu}$ et al [6]. Classification has been carried out only on areas with coarse texture to know more about local structure. Training artificial neural networks for pavement distress image compression, noise reduction and evaluation led to further studies and activities on the same technique [7][8]. Chambon et al [9] introduced a wavelet and 2D matched filter in order to define an adapted wavelet and then used the results of this multi scale detection into a Markov Random Field (MRF) process to segment fine structures of images for road crack detection.

Bi-directional Empirical Mode decomposition (BEMD) for edges detection is uti-lized by Prah et al [10]. Canny and Sobel methodologies have been compared with BEMD in their research. They conclude that different edge detection techniques are more efficient for several types of surfaces. Appreciable performance of Sobel meth-odology for Portland cement concrete and canny methodology for asphaltic concrete support the above conclusion. In another significant contribution, Oliviera and Cor-reia [11], have explored the area of crack detection and classification with a super-vised learning approach. In their research, the image database was divided into image subsets in order to handle the large amount of input data. They have introduced three parametric and three non-parametric supervised classification strategies to infer the cracks. Thereafter the cracks are classified into types like longitudinal, traversal and mixed by exploring their 2D geometric features.

Other directional line detection techniques such as matched and Gabor filter have also been used for crack detection in recent times. However, a thorough review of literature shows that neither Hough transform nor any of its variants are used for crack detection, either in pavement images or other surfaces such as concrete, ceram-ics, etc for which crack detection work is extensively found.

\section{FUZZY HOUGH TRANSFROM}




\section{Hough Transform}

The Hough transform is one of the methods used to find shapes from a set of points in a given space [12]. Hence, this is frequently used to find arbitrary shapes in an image. The detection is performed by a voting procedure. A binary image (the concept is also applicable to gray scale images) will be the result of some processing operation, such as thresholding, where the foreground and the background will be of contrasting val-ues in the original image. To detect lines in these images, the fact through any given pixel an infinite number of lines can pass is used. So, there is a chance that any of these lines can be common between a given number of pixels, forming the basis for a line detection. Referring to Figure 2, for an arbitrary pixel with coordinates ( $\left.\mathrm{x}_{0}, \mathrm{y}_{0}\right)$ in the image plane $\mathrm{xy}$, a line that goes through it is shown in green color.

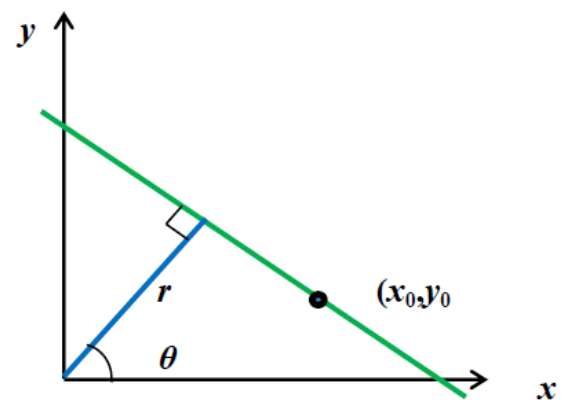

Figure 2. Definition of the $(r, \theta)$ pair for a line in the $x y$ plane.

The equation of any line, which is generally written in terms of the gradient of the line with the $x$ axis and its intercept on the $y$ axis, can also be written as a function of the $(r, \theta)$ plane as,

$$
y_{0}=\left(-\frac{\cos \theta}{\sin \theta}\right) x_{0}+\left(\frac{r}{\sin \theta}\right)
$$

Rearranging the equation, it can be shown that if, $\theta \in[0,2 \pi)$,

$$
r(\theta)=\left|x_{0} \cos \theta+y_{0} \sin \theta\right|
$$

Where, $r \in[0, D], D$ being the diagonal length of the image in number of pixels.

Equation 2 represents all the lines that go through a given point $\left(x_{0}, y_{0}\right)$ in the image. Moreover, it can be noted that the expression for $r$ in Equation 2 represents a sinusoid in the $(r, \theta)$ plane. So for every pixel detected in the image plane $x y$, there will be a sinusoid in the $(r, \theta)$ plane, representing all the lines going through it. The intersections of the sinusoids of a number of detected pixels is detected as a line. For practical reasons, both the $r$ and $\theta$ axes have to be discretized forming bins. These bins in the $(r, \theta)$ plane give the appearance of an image and are called the accumulator array.

\section{Fuzzy Hough Transform}

If the Hough transform for straight-line detection is applied to an image, the line seg-ments in it can be detected. However, the binary crack image shown in Figure 1 clear-ly shows that crack segments do not belong to straight lines, but are only nearly straight. Hence, the limitation of the Hough transform must be removed before it can be used to detect cracks. This is performed with the help of the fuzzy Hough trans-form. The original Hough transform, explained in the previous section, only considers the pixels that falls on a line. Whereas, the fuzzy Hough transform accommodates for the fact that there can be pixels slightly away from a given line (i.e. a bin in the $r-\theta$ accumulator array) due to sensor noise or due to any discrepancies in preprocessing operations such as thresholding or edge detection [13]. The closer the pixel to any line, hence the corresponding accumulator array bin, the more its contribution. This capability of the fuzzy Hough transform is utilized here to detect crack lines that are inherently non-straight. It has been shown [14] that the easiest way to get the fuzzy contribution of all pixels is to form the accumulator array with the usual Hough trans-form and then convolve it with the following 1-D kernel in the $\rho$ direction,

$$
g(d)=\left\{\begin{array}{lr}
e^{-\frac{d^{2}}{\sigma^{2}}} & \text { if } d<R \\
0 & \text { otherwise }
\end{array}\right.
$$


Where, $R=\sigma$, defining the width of the kernel. The larger the width of the kernel the more non-straightness the algorithm can tolerate. However, on the downside, more noise pixels can also get detected as crack segments. For smaller kernel widths noise detections will be reduced at the expense of detected crack segments.

\section{Tiled Hough Transform}

Dividing the image into a number of tiles reduces the contribution of noise/ texture pixels to the accumulator array bins. This is known as the tiled Hough transform [15].

In this paper, exploiting capabilities of both the methods for the first time, the tiled fuzzy Hough transform is used.

\section{METHODOLOGY}

The images used in this study are captured by the LRIS pavement imaging system manufactured by Pavemetrics Systems Inc., Canada [16]. The physical resolution of the road captured by the images is $1 \mathrm{~mm} /$ pixel. Each image is 1080 x 900 pixels in size. The algorithm is coded in MATLAB. First the image is binarized to use the Hough transform. A threshold value of 0.22 is used (see Figure 1). In addition, the image is divided into a number of tiles and the tiles are numbered. 40 x30 segments in the $x$ and $y$ directions result in 1200 tiles.. Now the image is inverted before it is skeletonized.

The images are eroded until the skeletons are single pixel in width. The reason for skeletonizing is to reduce the possibility of the pixels along the width of the image being detected as a crack segment. This is in keeping with the fact that most of the horizontal (i.e. transverse to the load length) cracks are very low in contrast, as seen in Figure 1. The contrast results in a low number of pixels for transverse cracks after binarization, and due to this fact the threshold value of the accumulator array cannot be very high. Once the skeletonization is done, the transverse cracks and the longitudinal (i.e. close to the vertical in Figure 1) one are brought closer when considering the number of pixels. Without this operation, there is a huge disparity between the pixels for the transverse and longitudinal cracks in the in the binarized image, leading to across-the-width detections for longitudinal cracks.

Choosing a $\rho$ resolution of 1 pixel and a $\theta$ resolution of $20^{\circ}$, an accumulator array is formed for each tile. For $\sigma=3$, the convolution kernel, in Equation (3), is calculated. Subsequently each of the accumulator arrays, 1200 in total, are convolved with the kernel along the $\rho$ direction. A threshold value of 10 is used for the accumulator ar-rays to detect the $\rho$ and $\theta$ values representing crack segments in each array. The $\rho$ and $\theta$ values, thus found, are then used to plot detected crack segments in the tiles.

\section{RESULTS}

Figure 3 shows the detection of a longitudinal crack with that of a transverse one which is barely seen. It can be observed that the longitudinal one is more prominent with a good contrast leading to a good detection. Even the sections of it that are very thin are properly detected, showing the strength of the proposed method. The reason for the transverse crack to be low in contrast is due to the fact that the artificial lighting of the LRIS system, and almost all of the other platforms for this purpose, is in the transverse plane. Given that cracks are thin channels, resembling trenches, the transversely pointed illuminators illuminate the transverse cracks all the way down to the bottom leaving hardly any contrast between them and the regular road surface. Whereas for the longitudinal cracks, the light rays from the transverse directional illumination is blocked by the side walls of the crack 'trenches' from reaching the bottom, resulting in dark image areas leading to a good contrast between the crack and the background. Some dark spots outside the crack area are also detected.

The detections on a near longitudinal crack show the power of the algorithm (see Figure 4). Here, even very thin tortuous sections toward the top-left hand corner of the image are detected accurately. No false detections are made. 

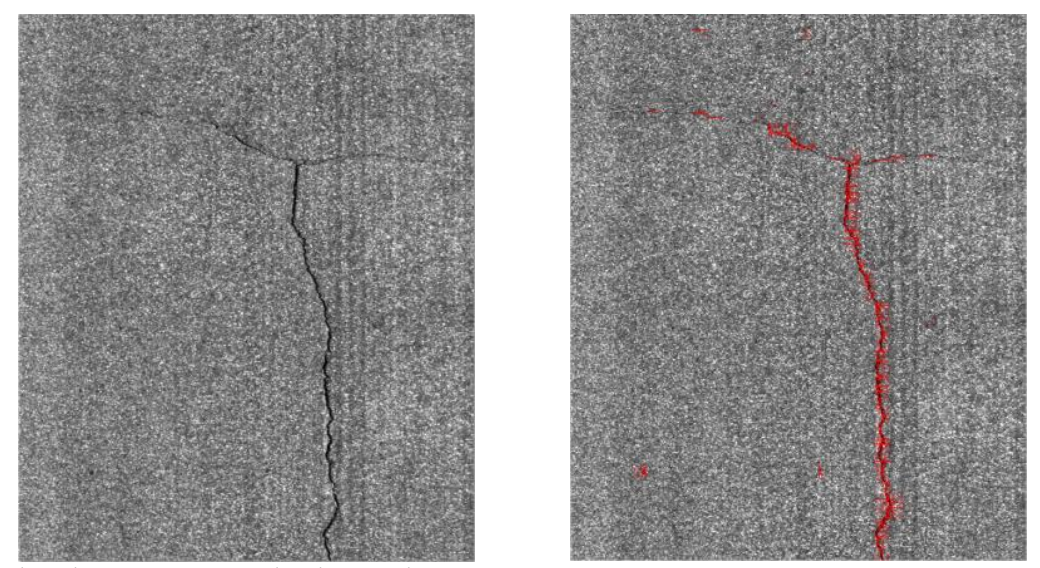

Figure 3. Longitudinal and transverse cracks detected.

$$
\begin{aligned}
\text { Precision } & =\frac{\text { True positives }}{\text { True positives }+ \text { False positives }} \\
\text { Recall } & =\frac{\text { True positives }}{\text { True positives }+ \text { False negatives }}
\end{aligned}
$$

A total of 20 images are tested for the algorithm. The length of the crack segments are measured with a ruler in a highly enlarged version of every image. The enlargement is kept constant for all 20 images. The length of the correctly detected (i.e. true positives) and falsely identified (i.e. false positives) are also measured. The false negatives for each image is calculated by subtracting the true positive crack length from the total crack length enabling the calculation of the precision and recall values of the detections using Equations 4 and 5. For the image shown in Figure 3, a precision value of $90 \%$ and a recall of $70 \%$ are obtained. The loss of the performance is mainly due to the transverse crack. For the one shown Figure 4 a precision and recall of $98 \%$ and $94 \%$, respectively, are obtained. These are exceptionally good values. Hence, if the hardware of the imaging platforms are modified to image transverse cracks as accurately as the longitudinal ones, the $98 \%$ and $94 \%$ performance can be expected from the algorithm. The overall precision for the image set is $78.5 \%$ and its recall is $71.6 \%$.

\section{CONCLUSION}

This paper used the tiled fuzzy Hough transform is utilized for the first time to detect pavement cracks. It is shown clearly that cracks are detected efficiently with the pro-posed method. A precision and a recall of $78.5 \%$ and $71.6 \%$ are obtained. For longitudinal crack segments showing a good contrast, the obtained values are in the mid to high 90 percentile.

\section{ACKNOWLEDGEMENT}

The permission of Pavemetrics Systems Inc., Canada to use their images is greatly acknowledged.

\section{REFERENCES}

[1] "Flexible Pavement Maintenance and Rehabilitation," in Australian Asphalt Pavement Association, 2010.

[2] Chambon, S., "Detection of Points of Interest for Geodesic Contours - Application on Road Images for Crack Detection," in VISAPP, 2011, pp. 210-213.

[3] Zou, C., Cao, Y., Li, Q., Mao, Q., and Wang, S., "CrackTree: Automatic crack detection from pavement images," in Pattern Recognition Letters 33, 2012, pp. 227-238.

[4] Cheng, H., and Miyojim, M., Automatic Pavement distress detection system, Journal of In-formation sciences, Vol.,108(July 1998), pp 219-240. 
[5] Cheng, H.D., Chen, J.-R., Glazier, C., and Hu, Y.G., "Novel Approach to Pavement Cracking Detection Based on Fuzzy Set Theory", Journal of Computing in Civil Engineer-ing, October 1991, Pp. 270 -280.

[6] Hu, Y., and Zhao, C. -X., "A Local Binary Pattern Based Methods for Pavement Crack Detection," Journal of Pattern Recognition Research 1, pp. 140-147, 2010.

[7] Saar, T., and Talvik, O., "Automatic Asphalt pavement crack detection and classification using Neural Networks," in 12th Biennial Baltic Electronics Conference (BEC), 2010, pp. 345-348.

[8] Subirats, P., Dumoulin, J., Legeay, V., and Barba, D., "Automation of Pavement Surface Crack Detection using the Continuous Wavelet Transform," in IEEE International Conference on Image Processing, 2006, pp. 3037-3040.

[9] Chambon, S., Subirats, P., and Dumoulin, J., "Introduction of a wavelet transform based on 2D matched filter in a Markov random field for fine structure extraction: application on road crack detec-tion", SPIE 7215. Image Processing: Machine Vision Applications II, 72510A, 02 Feb, 2009.

[10] Prah, A.A., and Okine, N.A., "Evaluating pavement cracks with bi dimensional empirical mode decomposition," EURASIP J. Adv. Signal Process, vol. 1, pp. 861701-1-861701-7, 2008.

[11] Oliveira, H., and Correia, P. L., "Supervised Crack Detection and Classification in Images of Road Pavement Flexible Surfaces," in Recent Advances in Signal Processing, A. A. Zaher, Ed. 2009.

[12] Sonka, M., Hlavac, V. and Boyle, R., 1999. Image Processing Analysis, and Machine Vi-sion. 2nd Edition, PWS Publishing.

[13] Jaime-Castillo, S., Medina, J.M., Garrido, A.: A Method for Locating the Iliac Crests Based on the Fuzzy Hough Transform. ;In IFSA/EUSFLAT Conf.(2009)1063-1067

[14] Joon, H.H., Kóczy, L.T., and Poston, T. (1994).“Fuzzy Hough Transform”. Pattern Recognition Letters 15, pp. 649658.

[15] Jung, C. R., and Schramm, R., Parallelogram detection using the tiled hough transform. In Proc. of Intl. Conf. on Systems, Signals and Image Processing, pages 177-180, 2006.

[16] Pavemetrics LRIS. Accessed on 2 December 2014, from http://www.pavemetrics.com/en/lris.html 